\title{
Germanica
}

\section{La réception du roman Ein weites Feld de Günter Grass en Allemagne}

Joseph Jurt

\section{OpenEdition}

1 Journals

Édition électronique

URL : http://journals.openedition.org/germanica/2330

DOI : 10.4000/germanica.2330

ISSN : 2107-0784

Éditeur

Université de Lille

\section{Édition imprimée}

Date de publication : 30 décembre 1999

Pagination : $37-47$

ISBN : 9782913857018

ISSN : 0984-2632

Référence électronique

Joseph Jurt, "La réception du roman Ein weites Feld de Günter Grass en Allemagne », Germanica [En ligne], 25 | 1999, mis en ligne le 28 janvier 2014, consulté le 06 octobre 2020. URL : http:// journals.openedition.org/germanica/2330 ; DOI : https://doi.org/10.4000/germanica.2330

Ce document a été généré automatiquement le 6 octobre 2020.

(c) Tous droits réservés 


\title{
La réception du roman Ein weites Feld de Günter Grass en Allemagne
}

\author{
Joseph Jurt
}

«Le chemin menait tout droit à l'unification allemande, sans que les élites intellectuelles de RDA y jouent dorénavant un rôle déterminant ", écrit Anne-Marie Corbin-Schuffels au sujet des événements de novembre $1989^{1}$. Le constat rejoint celui exprimé par Wolf Lepenies dans son petit livre Aufstieg und Fall der Intellektuellen in Europa ${ }^{2}$. Il parle des intellectuels est-allemands comme de " héros de cinq jours », c'està-dire entre le 4 et le 9 novembre 1989. Lors de la grande manifestation du 4 novembre à l'Alexanderplatz, des intellectuels, notamment Christa Wolf et Heiner Müller, demandèrent le droit à la parole, mais la chute du Mur, le 9 novembre, fut le résultat de la pression populaire. La première révolution qui avait réussi en Allemagne a été, ainsi que le souligne Lepenies, l'effet d'une levée en masse, mais ni la conséquence d'un affrontement long et conséquent, comme ce fut le cas en Pologne avec le mouvement Solidarité, ni le triomphe de vingt ans de résistance comme à Prague ou à Bratislava. Le changement a été imposé par les milliers de personnes fuyant le régime dictatorial, qui avaient trouvé refuge dans les ambassades allemandes de Prague et de Budapest, et par tous ceux qui manifestaient dans la rue à Leipzig et à Dresde. Les intellectuels avaient cru voir s'exprimer dans le slogan « Nous sommes le peuple » la réalisation d'un rêve socialiste, mais les masses lui préférèrent "Nous sommes un peuple » pour souligner leur désir d'unification. "Écrivains et intellectuels avaient perdu leur fonction de soupape de sécurité en RDA $»^{3}$. La RDA a été, comme l'affirme Wolf Lepenies, le seul pays de l'Europe de l'Est où il y avait une scission entre le peuple et l'intelligentsia ${ }^{4}$. Les intellectuels en RDA, loin d'être marginalisés et forcés de travailler comme ouvriers, ont joui de beaucoup de privilèges. À l'exception d'Erich Loest, il y eut peu de dissidents victimes et les victimes comme Wolfgang Harich et Walter Janka ne se sont pas considérées comme des dissidents. L'exil en RFA n'a pas été contraignant puisqu'il s'inscrivait dans la même aire culturelle et linguistique. 


\section{Günter Grass et l'unification allemande}

2 Si les intellectuels est-allemands n'ont pas joué un rôle moteur lors du processus d'unification, ils ne pouvaient éviter d'interpréter cet événement qui a profondément modifié le profil de l'Allemagne, de même que leurs collègues ouest-allemands. C'est notamment Günter Grass qui est intervenu, à maintes reprises, en tant qu'intellectuel, défendant une position qui ne s'insérait pas purement et simplement dans le mainstream. Grass a été marqué par sa jeunesse à l'époque du Troisième Reich : «Il n'a jamais oublié ni son enrôlement forcé dans les jeunesses hitlériennes, ni la visite d'un camp de concentration qu'on lui imposa pour l'édifier, le dessiller $»^{5}$. À l'instar de Heinrich Böll et de Siegfried Lenz, Günter Grass était devenu une sorte de conscience nationale. "Les sujets de mes livres m'ont toujours paru dictés par l'histoire de l'Allemagne ", remarqua-t-il dans un entretien en $1995^{6}$. De par son talent, il serait plus attiré par le côté artistique. En Allemagne, il avait dû apprendre qu'une forme vide de contenu ne valait rien. Dès ses premières œuvres, il s'était aussi confronté à la réalité contemporaine immédiate.

Grass s'est ainsi exprimé dès 1989 au sujet du processus d'unification 7 . Il ne s'y est pas opposé, mais a regretté qu'il soit entrepris à la hussarde, sans concertation. Il a relevé les traits pré-modernes de la société est-allemande qu'il caractérisait par un rythme de vie plus lent, alors que l'unification s'est caractérisée par l'accélération, voire la précipitation. Grass s'était notamment élevé contre la métaphore dominante proposée par Rudolf Augstein dans l'hebdomadaire Der Spiegel, celle du train qui était parti et qu'il fallait prendre de vitesse ${ }^{8}$. Grass avait plaidé pour une confédération des deux États et non pas pour une simple unification: une assemblée constituante aurait dû alors élaborer une nouvelle constitution'. Selon l'article final de la Loi fondamentale (art. 146), il aurait fallu - après un véritable débat - proposer une nouvelle constitution au peuple allemand. Or, c'est la simple adhésion de l'ancienne RDA qui a été retenue, aux yeux de Grass une violation de la Constitution. "Il faudra le payer un jour ou l'autre ", déclara l'écrivain dans un entretien accordé au Monde ${ }^{10}$. Pour Grass, il ne devait plus y avoir d'Allemagne réunifiée après Auschwitz. Il redoutait le danger d'une "Grande Allemagne ", centralisée de plus en plus autour de Berlin - à la fois ancienne et nouvelle capitale - à laquelle il aurait préféré Francfort. «L'unité a toujours bousillé la démocratie ", fait-il dire à l'un des personnages de son roman Ein weites Feld. Une telle affirmation n'est pas totalement exacte, car les partisans du fédéralisme adhéraient, au siècle passé, au particularisme de l'Ancien régime alors que les militants de l'unité nationale luttèrent en même temps pour la liberté et la justice. Grass se battait surtout pour qu'on respectât davantage les traditions et le passé de deux générations qui avaient dû vivre en RDA : "J'avais espéré qu'on se montrerait plus respectueux envers ces seize millions de personnes qui ont supporté le plus lourd fardeau de la guerre, qui, pour les anciens, étaient passés sans transition d'une dictature à une autre, du nazisme à la dictature stalinienne ; qu'on serait moins brutal, que l'on respecterait leurs quarante années passées dans une société fermée. Or rien de tout cela n'a été pris en considération $»^{11}$.

Dans un entretien accordé à Ingeborg Villinger cinq ans après la chute du Mur, Günter Grass se disait assez déçu par l'immobilisme des hommes politiques, qui aurait encore renforcé les tendances négatives. Il avait lui-même cessé d'intervenir directement pour faire face à ce présent immédiat par le biais d'un grand roman, Ein weites Feld. Après sa 
parution en août 1995, cette œuvre a suscité un débat d'une extrême violence, qui a largement dépassé le monde clos de la critique littéraire.

\section{La création d'un événement}

Les réactions massives au roman Ein weites Feld en dehors des milieux littéraires (la maison d'édition a recensé plus de 10000 réactions ${ }^{12}$ ) sont de bon augure. Le roman aurait réussi à faire que les citoyens soient confrontés au présent et au passé de l'Allemagne. En effet, des hommes politiques se sont exprimés sur le roman, un fait extrêmement rare en Allemagne. Or, ce n'est pas le roman qui a suscité ces interventions. Ce furent plutôt des réactions aux réactions - un débat secondaire s'instaurant à propos du livre ou en dehors de lui. Les réactions des politiques ont été provoquées par une remarque de l'écrivain qui disait avoir voulu montrer par des moyens littéraires les motifs de l'assassinat de Detlev Rohwedder, l'ancien patron de la Treuhandanstalt (organisme chargé de privatiser les entreprises de l'ex-RDA), par un commando de la Fraction Armée rouge. Peu après, Birgit Breuel, l'ancienne présidente de la Treuhand, se montra indignée que de telles légendes puissent naître et le ministre fédéral des Finances, Theo Waigel, s'associa à ses reproches lors d'un meeting de son parti à Munich. Enfin, une représentante des Verts, familière des talk-shows, Antje Vollmer, intervint dans Die Zeit, le 8 septembre 1995, pour déplorer que la violence entre intellectuels ne soit guère un modèle pour les jeunes. Tous les politiques se sont servis du débat non pas pour traiter du livre, mais pour se manifester dans un contexte propice à des interventions très générales.

6 Si le livre - ou plutôt l'affaire Grass - connut une telle résonance, c'est qu'il était devenu un "événement ", critère nécessaire pour que les médias « couvrent » un fait. Or l'événement est, en même temps, créé par les médias. Dans ce contexte, il faut mentionner trois faits qui contribuèrent à la création de l'événement médiatique : tout d'abord la campagne de lancement opérée par la maison d'édition, ensuite la critique virulente publiée par l'hebdomadaire Der Spiegel et enfin l'émission télévisée Das literarische Quartett.

7 Il y eut tout d'abord la campagne de lancement au compte-gouttes organisée par le nouvel éditeur de Grass, Steidl. Depuis 1993, Grass avait quitté sa traditionnelle maison d'édition Luchterhand (qui avait changé de main) pour céder tous ses droits à celle que dirigeait à Göttingen Gerhard Steidl. Dans le domaine de la littérature, c'est Grass qui occupe, avec un tirage global de 20 millions d'exemplaires, la première place. En éditant le "grand roman de l'Allemagne ", l'éditeur espérait réussir un grand coup. Il prépara une campagne de lancement particulièrement " professionnelle », trop peutêtre, pour parvenir au succès escompté. Au début de l'année 1995, l'écrivain accorda un entretien à la Süddeutsche Zeitung où il évoquait le roman qu'il était sur le point de terminer. Début mars, les agences annoncèrent que l'écrivain était en train de corriger ses épreuves. Le 25 avril, il lut pendant une heure et demie des extraits de son roman au Centre de la communauté juive à Francfort, invité par Marcel Reich-Ranicki, le «pape » de la critique littéraire en Allemagne, qui avait auparavant éreinté toutes ses œuvres. Mais ce soir-là, Reich-Ranicki exprima son admiration à propos des passages qu'il venait d'entendre. La sortie du livre était prévue pour le 28 août et des journalistes remarquèrent que cette date était celle de la naissance de Goethe - autre mégalomanie - alors que la maison d'édition avait opté pour ce lundi pour des raisons d'ordre 
pratique. En même temps, la presse fut priée de ne pas parler du livre avant le jour de sa sortie. À travers cette campagne promotionnelle savamment orchestrée, la maison d'édition avait su créer un véritable suspense, mais elle suscitait aussi une certaine méfiance, la campagne trahissant une trop grande habileté commerciale, ce qui n'est guère apprécié dans le monde de l'édition littéraire. Si certains chroniqueurs commentèrent la campagne sur un mode quelque peu ironique ("Grassomanie », " en attendant Grass »), l'éditeur avait pourtant réussi un coup médiatique. On ne cessa de parler du roman tant attendu de la réunification allemande.

Cependant, en lieu et place des «bonnes feuilles", le Spiegel publia le 21 août une critique cruelle du roman. Marcel Reich-Ranicki, n'ayant pu publier son compte rendu dans « son » ancien journal, la $F A Z$, c'est le Spiegel qui lui offrit une tribune, ce qui n'a pas laissé d'étonner. La métaphore du déchireur de livre (en allemand, jeu de mots par analogie avec "éreinteur ») a été réactivée de nouveau à la une du Spiegel du 21 août 1995 avec un montage qui montrait Reich-Ranicki en colère déchirant effectivement le nouveau livre de Grass. Cette image, qui imitait le geste du Moïse de Michel-Ange, eut l'heur de plaire au critique puisqu'elle allait faire monter le tirage de l'hebdomadaire. Ce qui a pu paraître particulièrement cynique, c'est que l'intellectuel juif allemand le plus connu en Allemagne se soit prêté à ce jeu, même si la photo n'entendait pas inciter à des autodafés. Le compte rendu du livre fut écrit par Reich-Ranicki dans le Spiegel sous forme de «lettre ouverte » qui suggérait une intimité entre le critique et l'auteur, certes inexistante, mais qui permettait d'adopter un ton condescendant. Après un effet de manche (Grass, "un écrivain hors du commun, plus encore, que j'admire»), le verdict était sans appel: "Je trouve votre roman Ein weites Feld complètement raté, désastreux ». Et le critique avançait une explication psychologisante, plutôt humiliante. Grass ne souffrirait pas de mégalomanie, comme certains l'affirmaient, mais plutôt d'un manque de confiance en soi qui aurait paralysé sa force créatrice.

9 La méthode de Reich-Ranicki est extrêmement simple : il part de la vieille équation «l'homme et l'œuvre». De plus, il n'admet qu'une seule norme poétologique pour le genre épique, la narration. Un auteur doit vivre le présent et le faire vivre à la manière réaliste des premiers romans de Grass - argument qui reviendra dans d'autres comptes rendus. Le narratif apparait comme le seul mode romanesque, le romanesque discursif qui distingue le roman moderne étant exclu. Et puis, il y a pour Reich-Ranicki une autre norme d'appréciation : un contenu politiquement correct. Il reproche à Grass de ne pas s'indigner dans son livre de la répression qui existait en Allemagne de l'Est. Le critique semble s'inspirer ici de l'héritage poétologique du réalisme socialiste où la littérature est constituée par un contenu substantiel et un héros positif !

Ce type de critique sommaire a été encore plus visible lors de l'émission télévisée du 24 août, Das literarische Quartett, sorte d'Apostrophes dirigée par le même Reich-Ranicki et très suivie à cause des charges féroces qu'il orchestre avec trois collègues. ReichRanicki réitéra son verdict brutal devant les caméras : un livre illisible et sans valeur. On ne saurait rendre la vie à ce qui était mort. Grass aurait souhaité une critique littéraire compréhensive qui expliquait une œuvre et ne se considérait pas comme un tribunal. Reich-Ranicki rétorqua que l'écrivain avait une conception de la critique analogue à celle de Goebbels, ministre de la Propagande nazie, pour qui de simples « rapports » devaient remplacer les critiques littéraires, genre perverti par les auteurs juifs comme Heinrich Heine ou Alfred Kerr; le pouvoir politique était la seule compétence en matière de jugement. Cette association a paru particulièrement ignoble 
face à un Grass antifasciste et constamment soucieux de ne pas tirer un trait sur le Troisième Reich.

11 La critique littéraire allemande passe, notamment en France, pour moins « corruptible » parce que le « métier» de critique y exclut le cumul des fonctions. Mais l'idée de l'équité d'une critique littéraire plus indépendante est un mythe. Une critique systématiquement négative rend aussi peu justice à un livre qu'une critique de complaisance. Ce qu'il faut d'abord - et là on ne peut qu'approuver Grass - c'est comprendre le livre à partir de sa logique interne. L'exécution d'un livre, qui peut être justifiée, sert souvent au critique à se mettre en valeur.

\section{La tendance des jugements}

À travers tel ou tel éreintement célèbre se crée aussi la légende d'un livre. Hellmuth Karasek, participant à la célèbre émission Das literarische Quartett, affirme ainsi que la critique allemande considérait unanimement le livre de Grass comme un échec. Or un examen plus détaillé des comptes rendus permet de nuancer beaucoup cette affirmation à l'emporte-pièce. Sur les 40 premiers comptes rendus que j'ai pu lire, seuls 21 ont été entièrement négatifs, 6 émettaient des réserves et 13 ont été positifs. Les échos de la presse régionale, notamment de l'Ouest, ainsi que ceux de l'Allemagne de l'Est $^{13}$ ont été plus positifs. Dans les colonnes de Die Zeit, le 25 août 1995, Iris Radisch a considéré le roman comme "illisible", mais elle lui a tout de même consacré deux grandes pages. Le jugement de Reinhard Tschapke dans Die Welt a été tout aussi lapidaire : «C'est le livre le plus épais et le plus léger de Grass».

Beaucoup de jugements sont émis à partir d'un horizon d'attente implicite auquel le roman ne correspondait pas. On part de l'idée d'un écrivain représentatif qui devrait fournir le roman national représentatif. Selon Gustav Seibt de la $F A Z$ du 19 août 1995, on aurait considéré Grass à tort comme un nouveau Thomas Mann ayant la vocation de dresser un panorama complet de la nation. Le résultat - le roman - témoignerait des meilleures intentions de l'auteur, d'un labeur énorme et en même temps de l'absence de tout sens critique : bref, "un enfant mort-né, un monstre». Peter Wapnewski perçoit dans sa critique publiée par Focus, le 28 août 1995, l'intention de l'écrivain de nous donner une allégorie ambitieuse de la recherche utopique de l'unité allemande et de ses conséquences fatales, mais l'auteur n'en aurait pas réussi la transposition littéraire. D'autres critiquaient les longueurs, l'absence de suspense, le ton parfois trop académique (avec les allusions à l'histoire littéraire faisant le bonheur des seuls germanistes).

14 Mais il ne faut pas non plus négliger les jugements positifs qui ont été formulés au sujet du roman. Aux yeux du General Anzeiger de Bonn, le 28 août 1995, Grass a écrit le grand roman de notre temps. Le 24 août 1995, pour Wolfgang Ignée de la Stuttgarter Zeitung, Grass a réussi, d'une manière convaincante, le grand roman de l'unification allemande, attendu depuis longtemps. Jürgen Busche parle dans les colonnes de la Süddeutsche Zeitung, le 24 août 1995, d'une "esquisse ambitieuse de la topographie de l'âme allemande entre politique et littérature ». Les jugements positifs se fondent en premier lieu sur des critères littéraires. La critique du Hamburger Abendblatt du 21 août 1995 admire la grande force stylistique de l'auteur ainsi que la densité de l'évocation des paysages berlinois chers à Fontane. Ce que la critique a surtout retenu, c'est la complexité de la structure et le message politique implicite du roman. 


\section{Une structure complexe} de l'Allemagne contemporaine, d'abord dans la Maison des ministères de la RDA, puis au siège de la Treuhand. Fonty est une réincarnation de l'écrivain Fontane dont il sait tout, ce qui permet à Grass de mettre constamment en parallèle deux figures, le créateur et son sosie ; et deux époques, celle de la première unification sous Bismarck, et l'autre sous Kohl. Wuttke se distingue par son caractère médiocre et parfois incohérent, n'étant ni héros, ni résistant, ni collaborateur. Il est constamment accompagné par Hoftaller, une réincarnation de l'agent éternel et, plus concrètement, de la figure de Tallhover, issu du roman d'un autre auteur allemand, Hans-Joachim Schädlich. Il y a donc à deux reprises une double identité. « La relation entre Fonty et Hoftaller, ce mouchard de profession, est très ambivalente », remarque Grass. « C'est un chapitre sans fin, cette relation entre la littérature et la censure; et cette façon dont le censeur, le mouchard, doit se laisser imprégner par la littérature, en faire cas; il développe un amour, certes singulièrement déformé pour la littérature, mais un amour quand même. Fonty, l'écrivain, est à la fois observé et choyé. C'est une relation à double sens qui n'a rien des imageries manichéennes. C'est toute une trame de fils gris, une palette de nuances $»^{14}$.

Cette complexité du roman a donné du fil à retordre aux critiques. Pour Gustav Seibt de la $F A Z$, le 19 août 1995, l'idée de la duplication de la figure centrale aurait pu donner matière à un petit récit ou à une nouvelle selon le modèle de Treffen von Telgte, publié par Grass en 1979. Cet avis est partagé par Andreas Isenschmid dans l'hebdomadaire zurichois Die Weltwoche du 17 août 1995. Ein weites Feld, voilà « 200 pages excellentes dans 500 pages de matière grasse ». L'extension du livre à 150 ans d'histoire allemande ne serait pas évidente. Faut-il un roman sur toute une époque ? Existe-t-il un roman de la Révolution française ? Pour Peter Wapnewski, le 28 août 1995, dans Focus, l'essence d'un roman, c'est l'itinéraire linéaire d'une vie d'homme et non pas le panorama d'une époque. Jürgen Busche constate à son tour dans la Süddeutsche Zeitung du 19 août 1995 que le roman de Grass ne se déroule pas selon le schéma de l'intrigue linéaire; il y a plutôt une suite de scènes isolées dont l'évocation rappelle la peinture de genre. Selon ce même critique, la qualité esthétique du roman ne réside pas dans la cohérence de l'action, mais dans la densité du style. Grass a rappelé lui-même le modèle du récit picaresque, récit à épisodes, qui ne se caractérise pas par l'introspection psychologique, mais par une perspective extérieure sans hauteur de vue.

Si un certain nombre de critiques ont reproché à l'écrivain de ne pas avoir réduit l'idée de la duplication de la figure à un petit récit, d'autres approuveraient un long roman à condition qu'il emprunte à l'ample réalisme des premiers romans de Grass. Or, ici on resterait sur sa faim; au lieu d'un récit plein de vie, on trouverait un débat d'idées, dépourvu d'action. Wolfram Schütte objecte, cependant, dans sa critique publiée par la Frankfurter Rundschau du 26 août 1995 que le modèle du récit réaliste est ici une fausse clé; depuis le récit Treffen von Telgte, Grass s'est orienté vers un mode narratif aréaliste, plutôt maniériste. L'auteur décrit les manières de décrire. Son roman est un montage de citations historiques et contemporaines ; à travers la grille de l'histoire, il nous donne une copie ironique du présent; au lieu de traiter de la réalité de l'unification, c'est un roman sur les discours portant sur les événements historiques, 
sur les idées reçues à la manière de Bouvard et Pécuchet, modèle dont Grass se réclame explicitement. Le reproche adressé à ses personnages d'être des figures artificielles tombe à pic. L'auteur les a voulues ainsi. Elles sont des constructions, des marionnettes. Autres reproches: les répétitions, les reprises, l'exhaustivité des descriptions. Or, ceci s'explique à partir de la perspective narrative qui n'est pas individuelle, mais collective. Le roman commence par « Nous, ceux des archives ». L'écrivain Hermann Kant a trouvé géniale cette idée de la perspective collective, car elle permet de démontrer par le récit même l'obsession de l'observation exhaustive exercée par la Sûreté est-allemande.

\section{La dimension politique}

Beaucoup de critiques reprochaient à Grass d'avoir donné, dans son roman, une image anodine de l'ancienne RDA et d'avoir établi un parallèle entre la première et la deuxième unification allemande, conduisant à lire le roman comme un rapport historique. On blâmait Grass de ne pas avoir réussi la transposition romanesque et d'avoir instrumentalisé ses figures afin de divulguer ses opinions politiques personnelles, marquées par un conservatisme de gauche. On ne peut s'empêcher de penser, estimait Sigrid Löffler, dans le quotidien viennois Die Presse du 31 août 1995, que le texte littéraire sert de prétexte aux critiques pour condamner les opinions politiquement peu correctes de Grass au sujet de l'unification allemande. Le roman d'un auteur qui ne se résignerait pas à la situation inéluctable des faits et qui rêverait encore de la troisième voie d'un socialisme réformiste ne saurait être que ceci : « Le cul-de-sac de Bitterfeld » (in Die Zeit, le 25 août 1995). Le scandale résidait dans le fait que Grass, qui avait critiqué ouvertement la RDA, s'en prenait maintenant à la «Grande Allemagne ». Andreas Isenschmid, qui avait condamné le livre le 21 août 1995 dans Die Weltwoche, voyait en Grass le « dernier intellectuel». « Ce qu'on exige maintenant, c'est le consensus national qui a du mal à tolérer des opinions divergentes ", écrivait Manfred Bissinger dans Die Woche du 25 août 1995. «Jadis, oui, jadis, il y avait des débats, des confrontations, des camps distincts. Aujourd'hui règne le mainstream du showbiz public ». Et Grass estimait que son œuvre se devait d'être une voix adverse, une correction littéraire indispensable face à la version gouvernementale de l'histoire qui était en train de s'imposer nonobstant toutes les fautes et tous les manquements.

À juste titre, Grass a reproché à ses critiques d'identifier telle ou telle opinion d'une de ses figures avec celle de leur auteur et de ne pas respecter ainsi l'autonomie des personnages romanesques - point fondamental pourtant de toute lecture littéraire. Si le protagoniste Fonty est sceptique à l'égard de la réunification, sa petite-fille Madeleine, de surcroit française, approuve l'idée de la nation que la jeune génération doit édifier. Les points de vue se relativisent mutuellement et l'auteur n'est pas telle ou telle figure, mais la somme de toutes les voix.

En passant en revue les appréciations formulées par la plupart des grands critiques allemands au sujet du roman de Grass, on est étonné de voir combien ils se sont référés aux critères d'un horizon d'attente conservateur. D'une part, ils attendaient une confirmation affirmative du processus politique de l'unification, d'autre part, ils espéraient un roman réaliste, un bref récit linéaire ou une simple biographie de 
Fontane et des personnages en chair et en os. Ne tolérant pas une position politique critique, ils ne pouvaient être sensibles non plus aux innovations formelles. Ou bien, ayant condamné la position politique de l'auteur, ils devaient également refuser ses innovations formelles.

Günter Hoffmann, qui n'était pas non plus convaincu de la valeur artistique du roman, ne manquait pourtant pas de réfléchir dans Die Zeit du 25 août 1995 à l'importance de Grass en tant que personnage public: "Dans la République unifiée, on ne cesse de demander où est passée la voix des intellectuels. Mais, malheur à eux s'ils s'immiscent pour de bon dans les affaires politiques. Surtout s'ils le font en émettant une opinion « erronée ». Bien sûr, les politiciens et les intellectuels ne se barricadent plus dans le face à face acharné d'autrefois [...]. Mais il n'y a pas véritablement de dialogue, tel qu'il aurait pu accompagner, par exemple, le processus de réunification allemande, avec ses dimensions culturelles et psychologiques. Ce dialogue-là a échoué avant même de commencer ». Ce sont deux ou plusieurs Grass qu'il faut pour la République, conclut Hoffmann. «La future République de Berlin profitera toujours plus de cette attitude-là que de celle consistant à observer, à prononcer des interdits et, pour le reste, à se fier à Greenpeace ».

\section{NOTES}

1. Anne-Marie Corbin-Schuffels, La Force de la parole. Les intellectuels face à la RDA et à l'unification allemande (1945-1990), Villeneuve d'Ascq, Presses universitaires du Septentrion, 1998, p. 195.

2. Wolf Lepenies, Aufstieg und Fall der Intellektuellen in Europa, Francfort/New York, Campus, 1992, p. 56-61.

3. Anne-Marie Corbin-Schuffels, La Force de la parole, op. cit., p. 195 ; voir aussi Wolfgang Jäger/ Ingeborg Villinger, Die Intellektuellen und die deutsche Einheit, Fribourg, Rombach, 1997.

4. Voir aussi André Glucksmann : «La faille est ancienne, pas plus en Allemagne de l'Ouest qu'en Allemagne de l'Est, il n'y eut de Soljenitsyne et de Sakharov pour dénoncer, du temps de sa splendeur, l'archipel de la Stasi, pour en détailler les rouages et les parages. On connut à Berlin des oppositionnels et des contestataires fort honorables, mais pas de pensée dissidente comme à Moscou, Varsovie et Prague » (in Le Figaro, 2 octobre 1990, p. 4).

5. Angelo Rinaldi, L'Express, 25 septembre 1997.

6. «Ich will mich nicht auf die Bank der Sieger setzen », entretien avec Günter Grass, in $F A Z$, n²33, 7 octobre 1995.

7. Ses interventions ont été recueillies dans plusieurs volumes: Deutscher Lastenausgleich. Wider das dumpfe Einheitsgebot, Francfort/Main, Luchterhand Verlag, 1990 ; Rede vom Verlust. Über den Niedergang der politischen Kultur im geeinten Deutschland, Göttingen, Steidl-Verlag, 1992; Ein Schnäppchen namens DDR. Letzte Reden vorm Glockengeläute, Göttingen, Steidl-Verlag, 1993.

8. Voir à ce sujet Jürgen Link, «La réunification allemande comme événement symbolique », in Actes de la recherche en sciences sociales, ${ }^{\circ} 98$, juin 1998, p. 59-61, et dans le même numéro Joseph Jurt, « La nouvelle Allemagne : quels symboles?», p. 45-58.

9. Voir l'entretien accordé par Grass à I. Villinger dans Wolfgang Jäger/Ingeborg Villinger, op. cit., p. 233-234. 
10. Propos recueillis par Pierre Deshusses, Le Monde [des livres], 3 octobre 1997.

11. Le Monde, 3 octobre 1997 ; voir aussi Anne-Marie Corbin-Schuffels, op. cit., p. 225-226.

12. Une grande partie de ces réactions a été recueillie dans un volume publié par la maison d'édition de Grass : Oskar Negt (Ed.), Der Fall Fonty. 'Ein weites Feld' von Günter Grass im Spiegel der Kritik, Göttingen, Steidl-Verlag, 1996.

13. Voir aussi la remarque de Grass : «Certains critiques, notamment dans la presse de l'Est, ont souligné qu'il était surprenant de voir comment un homme de l'Ouest comme moi [...] était en mesure de mener un récit à l'intérieur de cette RDA en plein naufrage, en me plaçant dans la perspective de ceux qui étaient concernés. C'est le métier de l'écrivain d'être capable de se plonger dans les mentalités, les situations, de faire un avec elles, comme s'il les avait lui-même vécues » (Entretien accordé au Monde, 3 octobre 1997).

14. Entretien accordé au Monde, 3 octobre 1997.

\section{RÉSUMÉS}

Si les intellectuels est-allemands n'ont pas joué un rôle moteur lors du processus d'unification, ils ne pouvaient éviter d'interpréter cet événement qui a profondément modifié le profil de l'Allemagne, de même que leurs collègues ouest-allemands. C'est notamment Günter Grass qui est intervenu, à maintes reprises, en tant qu'intellectuel, défendant une position qui ne s'insérait pas purement et simplement dans le courant dominant.

Le présent article fait une synthèse des critiques formulées à l'occasion de l'événement que représenta la parution du roman de Günter Grass, Ein weites Feld. Certaines s'attachent à souligner la structure complexe de l'œuvre, mais c'est surtout sa dimension politique qui est l'objet des attaques, car Grass s'en prend à l'Allemagne réunifiée.

Haben die ostdeutschen Intellektuellen eine unwesentliche Rolle im Laufe des Vereinigungsprozesses gespielt, so konnten sie jedoch nicht umhin, dieses Deutschland so stark verändernde Ereignis zu interpretieren. Günter Grass vor allem hat als Intellektueller mehrmals das Wort ergriffen und dabei eine Haltung vertreten, die der vorherrschenden Geisteshaltung nicht ganz entsprach.

Der vorliegende Artikel ist eine Synthese aus den Kritiken, die anläßlich des durch die Veröffentlichung von Grass'Roman, Ein weites Feld, geschaffenen Medienereignisses verfaßt wurden. Manche betonen zwar, wie komplex einem die Struktur des Romans vorkommen mag, für die meisten wird aber dessen politische Dimension zur Zielscheibe massiver Angriffe, denn Grass stellt das vereinigte Deutschland in Frage.

\section{AUTEUR}

\section{JOSEPH JURT}

Universität Freiburg im Breisgau 